\title{
ㄷำ1
}

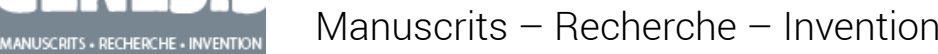

$34 \mid 2012$

Brouillons des Lumières

\section{Voltaire et le don du manuscrit}

\section{Nicholas Cronk}

\section{OpenEdition}

\section{Journals}

Édition électronique

URL : http://journals.openedition.org/genesis/897

DOI : 10.4000/genesis.897

ISSN : 2268-1590

\section{Éditeur :}

Presses universitaires de Paris Sorbonne (PUPS), Société internationale de génétique artistique littéraire et scientifique (SIGALES)

\section{Édition imprimée}

Date de publication : 10 avril 2012

Pagination : 19-36

ISBN : 978-2-84050-822-9

ISSN : $1167-5101$

\section{Référence électronique}

Nicholas Cronk, « Voltaire et le don du manuscrit », Genesis [En ligne], 34 | 2012, mis en ligne le 10 avril 2014, consulté le 13 juin 2020. URL : http://journals.openedition.org/genesis/897 ; DOI : https:// doi.org/10.4000/genesis.897 


\title{
Voltaire et le don du manuscrit
}

\author{
Nicholas Cronk \\ On parle beaucoup d' un nouveau poème de M. de Voltaire, intitulé Le Pauvre Diable. \\ Il n'est pas encore public. [...] Il a été apporté de Genève, moitié imprimé, moitié manuscrit. \\ Abbé Trublet à Jean-Jacques RousseAu 1
}

$\mathrm{U}$ n beau portrait de Marmontel, dû à Alexandre Roslin, montre l'auteur, coiffé d'un magnifique turban en soie, regardant vers le haut, d'un air inspiré ; dans ses deux mains il tient un manuscrit plié en plusieurs feuilles ${ }^{2}$. Le portrait porte la date de 1767, c'est-à-dire l'année de la parution de Bélisaire, livre qui lui attira une grande célébrité. Membre de l'Académie française depuis 1763 et déjà connu pour ses tragédies et ses Contes moraux, Marmontel aurait pu être dépeint tenant une de ses publications, selon une tradition bien établie ; mais il a préféré - le choix vient sûrement de Marmontel plutôt que de Roslin - se faire figurer avec un manuscrit, geste qui souligne l'acte d'écriture, instant unique qui capte le moment inspiré du génie. Le manuscrit nous rapproche du moment de la création, de l'acte même de créer.

Le monde littéraire depuis la Révolution est un monde de l'imprimé, dans lequel le manuscrit fait figure nécessairement de précurseur ; et implicite (quoique controversée) dans la notion d'étude génétique est l'idée finaliste d'une évolution du manuscrit vers l'imprimé. En ce qui concerne le XVIII siècle, la situation est différente dans la mesure où le monde du manuscrit coexiste avec le monde de l'imprimé. Beaucoup d'auteurs sous l'Ancien Régime préfèrent faire circuler un texte manuscrit plutôt que de le publier. Les relations entre l'imprimé et le manuscrit sont donc complexes et se présentent différemment pour chaque auteur. Quant à Voltaire, il offre une relation unique et particulière entre ce que François Moureau appelle la plume et le plomb ${ }^{3}$, et c'est de cette spécificité que je voudrais traiter ici. Je parlerai d'abord de la possibilité d'études génétiques de certains textes de Voltaire ; en deuxième lieu de Voltaire comme auteur de manuscrits ; et en dernier lieu des imprimés qui portent des touches manuscrites.

En 1779 , un an après sa mort, les papiers de Voltaire se trouvant à Ferney partirent avec sa bibliothèque à Saint-Pétersbourg, où ils se trouvent toujours. Un catalogue bref fut dressé rapidement par Caussy, avant la Grande Guerre ${ }^{4}$, et c'est maintenant une priorité de

1. D9003 (21 juin 1760). Les références « D » désignent Voltaire, Correspondence and related documents, éd. T. Besterman, Genève-Oxford, Voltaire Foundation, 1968-1977.

2. Le portrait est conservé au musée du Louvre (voir détail en couverture de ce numéro).

3. François Moureau, La Plume et le plomb. Espaces de l'imprimé et du manuscrit au siècle des Lumières, Paris, PUPS, 2006.

4. Fernand Caussy, Inventaire des manuscrits de la bibliothèque de Voltaire conservée à la Bibliothèque impériale publique de Saint-Pétersbourg, Paris, Imprimerie nationale, 1913.
Le manuscrit précède l'imprimé : l'étude génétique de textes voltairiens 
refaire ce catalogue, car les archives russes réservent certainement des surprises. D'autres manuscrits sont restés en France : certains sont bien connus ${ }^{5}$, d'autres ont disparu mais en laissant des traces dans des éditions imprimées. C'est notamment le cas avec l'édition de Kehl (1784-1789), préparée dans les années suivant la mort de Voltaire. Pour l'Épître à Daphné par exemple, poème adressé à Mlle Clairon, l'édition de Kehl est notre seule autorité pour une variante importante concernant Omer Joly de Fleury, ennemi acharné des philosophes. La variante en question est présentée ainsi : «On lit dans une autre copie $^{6} »$; il faut supposer que les éditeurs de Kehl avaient à leur disposition un manuscrit ( « une copie ») qui a disparu depuis. Voltaire, pas plus que ses contemporains, n'a gardé systématiquement ses manuscrits de travail, et parmi ceux qui restent, il y en a beaucoup qui relèvent du domaine théâtral, pour des raisons évidentes (la nécessité de multiplier les copies d'acteur, par exemple). Reste un certain nombre de cas où les manuscrits permettent de faire des études "génétiques », et je voudrais revoir ici le cas canonique de Candide.

À côté de la prolifération d'éditions imprimées, on ne connaît que deux manuscrits de Candide. Le manuscrit qui se trouve à la $\mathrm{BnF}$ est un simple fragment comprenant deux paragraphes du chapitre $22^{7}$. Le texte que nous y lisons correspond à l'édition Cramer de 1761, remaniée par Voltaire après la première édition de 1759 : le manuscrit est un document qui témoigne de la révision du texte par Voltaire, et relève donc seulement de la phase éditoriale de "l'avant-texte ». Un deuxième manuscrit, conservé à l'Arsenal, présente un cas très différent : il s'agit d'un manuscrit complet, envoyé par Voltaire au duc de La Vallière en 1758, année qui précède la première édition du roman ${ }^{8}$. Découvert par Ira Wade dans les années 1950, ce manuscrit énigmatique a fait l'objet d'études minutieuses, d'abord par Ira Wade 9 lui-même, ensuite par René Pomeau ${ }^{10}$ et par Frédéric Deloffre ${ }^{11}$, et je renvoie aux travaux de ce dernier qui font autorité.

Le manuscrit semble être en partie copié (au début), par une main inconnue, et en partie dicté par Voltaire, et corrigé de sa main. Dans la perspective d'une étude génétique, on peut signaler tout de suite deux grandes différences par rapport au texte imprimé. Sur la page de titre, on lit « Candide ou l'optimisme » mais non pas la suite, « traduit de l'allemand de Mr. le docteur Ralph » : cette évocation du rôle d'un traducteur - procédé romanesque s'il en est - semble être venue à Voltaire tardivement. Deuxièmement, on ne trouve pas dans le manuscrit de l'Arsenal, au chapitre 19, la célèbre rencontre avec l'esclave noir qui

5. Voir Andrew Brown (éd.), Calendar of Voltaire manuscripts other than correspondence, SVEC, $\mathrm{n}^{\circ} 77,1970$, p. 11-101.

6. Euvres complètes de Voltaire, 70 t., Kehl, Imprimerie de la Société littéraire typographique, 1784-1789, t. XIII, p. 189.

7. BnF, Ms. Rés. Z Beuchot 927 A.

8. Bibliothèque de l'Arsenal, ms. 3160. Le duc de La Vallière (1708-1780) était un militaire et un des grands bibliophiles du siècle. Une partie de sa bibliothèque célèbre fut acquise par le comte d'Artois et incorporée à la bibliothèque de l'Arsenal.

9. Ira O. Wade, «A manuscript of Voltaire's Candide », Proceedings of the American Philosophical Society, 101, $\mathrm{n}^{\circ}$ 1, 1957, p. 93-106 ; et « The La Vallière Manuscript », dans Voltaire and Candide: a Study in the Fusion of History, Art and Philosophy, Princeton, Princeton University Press, 1959, p. 157-180.

10. Voltaire, Candide, éd. René Pomeau, Paris, Nizet, 1959, p. 39-44.

11. Romans et contes, éd. F. Deloffre et J. Van den Heuvel, Paris, Gallimard, coll. « Bibliothèque de la Pléiade », 1979 , p. $836,841-853$. 
laisse Candide en larmes ${ }^{12}$. Il faut en même temps éviter de considérer que ce manuscrit est un texte «clos », rédigé d'un jet. "Le brouillon n'est pas une œuvre, nous rappelle Daniel Ferrer, mais un protocole opératoire en vue de la réalisation d'une œuvre13. » Une particularité du manuscrit de l'Arsenal consiste dans le fait qu'il contient deux versions du chapitre 22 (celui qui traite du passage de Candide et de Martin à Paris). Dans une analyse méticuleuse, F. Deloffre trouve deux états du texte à l'intérieur même de la première version du chapitre 22 , c'est-à-dire qu'il identifie trois états du texte en tout, ce qui lui permet, au moins pour ce seul chapitre 22 , de conduire une fine étude génétique qui nous conduit à regarder de près l'écriture de Voltaire.

La construction du manuscrit en tant qu'objet est aussi à étudier. André Magnan a récemment signalé l'existence d'une lettre ouverte de Beaumarchais qui apporte un nouvel élément à ce dossier :

Le feu duc de La Vallière [...] s'honora vingt ans d'être en relation avec Voltaire. Qu'on juge du plaisir que nous ressentions tous de lui voir arriver des montagnes de Suisse chaque chapitre de Candide, à mesure qu'ils s'échappaient de la tête de feu, rayonnante au milieu des neiges, avec les plaisants commentaires que l'auteur en faisait lui-même14 !

Nous savions que La Vallière connaissait le texte de Candide avant sa publication, et même que la duchesse de La Vallière avait écrit à Voltaire, en lui reprochant « tant d'indécence ${ }^{15}$ »; de la lettre de Beaumarchais nous apprenons en plus que ces envois furent accompagnés de «plaisants commentaires » de la part de l'auteur, c'est-à-dire d'invitations à La Vallière à lui répondre : plus qu'un simple état du texte, le manuscrit de l'Arsenal est une invitation au dialogue, et certaines des révisions apportées après ces échanges, aujourd'hui disparus, remontent peut-être aux suggestions du duc. Autre nouveauté, Voltaire aurait échelonné ses envois au duc (qui à cette époque habitait le château de Montrouge, au sud de Paris), un peu comme au XIXe siècle on donnait les romans en forme de feuilletons. Si l'on peut se fier à ce témoignage, et il n'y a pas de raison de ne pas lui faire confiance, il est lourd de conséquences pour notre lecture du manuscrit.

Sa construction est complexe et quelque peu énigmatique. Il est composé de petits cahiers de quatre pages (deux folios), reliés ensemble (ce qui n'est pas le format habituel de Voltaire dans un manuscrit de composition) ${ }^{16}$. À la fin de chaque cahier, une réclame annonce le premier mot du cahier suivant (comme dans bien des éditions imprimées), pratique qui vise à simplifier le travail du relieur. I. Wade, R. Pomeau et F. Deloffre traitent tous ce manuscrit comme s'il s'agissait d'un objet homogène et unique, et en effet, l'objet qu'on consulte aujourd'hui à la bibliothèque de l'Arsenal, relié de façon serrée, donne

12. Euvres complètes de Voltaire, Genève-Oxford, 1968-, [dorénavant OCV], t. XLVIII, p. 195-196 ; voir aussi p. 45-46 : R. Pomeau propose que cette addition tardive fut suggérée par la lecture de De l'esprit d'Helvétius à l'automne 1758 .

13. Logiques du brouillon. Modèles pour une critique génétique, Paris, Éditions du Seuil, 2011, p. 43.

14. André Magnan, «Une lettre oubliée de Beaumarchais sur l'édition de Kehl et sur la genèse de Candide », dans Voltaire en Europe : hommage à Christiane Mervaud, dir. M. Delon et C. Seth, Oxford, VF, 2000, p. 307-319 (à la page 307).

15. Voir D8072; et Romans et contes, éd. cit., p. 841.

16. Voir I. Wade, « The La Vallière Manuscript », op. cit., p. 159. 
l'impression d'être un seul manuscrit. Mais l'allusion aux chapitres qui arrivaient «à mesure qu'ils s'échappaient de la tête de feu » laisse clairement entendre qu'il y aurait eu une série d'envois. Il n'est pas possible que Voltaire ait envoyé chaque chapitre séparément : parfois les chapitres commencent au milieu d'une page, et même quand un chapitre commence en haut de la page, il ne se trouve pas au début d'un cahier. Il semble raisonnable de supposer que Voltaire n'aurait pas envoyé des cahiers séparés contenant seulement des parties de chapitres, et pour estimer donc le nombre d'envois, il faut identifier les cas où la fin d'un cahier coïncide avec la fin d'un chapitre. Une telle analyse donne le résultat suivant :

$\begin{array}{llll}\begin{array}{l}\text { Envois } \\ \text { (Ia) }\end{array} & \text { Chapitres } & \text { Pages } & \text { Réclame à la fin du dernier cahier } \\ \text { (Ib) } & 1-4 & \text { p. } 1-20 & \text { "Chap » } \\ \text { (II) } & 5-18 & \text { p. } 21-92 & \text { [aucune réclame] } \\ \text { (III) } & 19-22 \text { (i) } & \text { p. } 93-112 & \text { "chap. } 23 \text { » }\end{array}$

D'après cette analyse on peut déduire qu'il y a eu trois ou peut-être quatre envois. Le témoignage et la forme des réclames sont évidemment précieux. Le manque de réclame à la page 92, à la fin d'un cahier, semble confirmer que le copiste s'arrêta à ce point, sans connaître la suite. De même, la fausse réclame à la page 112 - le copiste écrit « chap. 23 » à la fin du chapitre 22, sans savoir que l'auteur allait réviser ce dernier chapitre - confirme sans doute aucun qu'il s'agit de la fin d'un envoi. Mais est-ce que la page 20 marque aussi la fin d'un envoi ? La réclame ici (« Chap ») n'est pas fausse, et c'est peut-être une simple coïncidence que la fin du chapitre vienne à la fin du cahier : dans ce cas, (Ia) et (Ib) ne font qu'un seul envoi, ce qui semble fort probable.

On voit ainsi que Voltaire a d'abord envoyé (en un ou deux envois) les dix-huit premiers chapitres (Ia et $\mathrm{Ib}$ ), qui étaient sans doute terminés (ce qui explique que dans cette première partie du manuscrit, le travail du copiste est peu corrigé). Ensuite il a envoyé les chapitres 19-22 (II), qui étaient en chantier, et qui sont plus corrigés. Peut-être que La Vallière a fait des commentaires sur ce chapitre 22, qui ironise sur la vie sociale parisienne, car le prochain envoi (III), qui contient le reste du roman, commence avec une nouvelle version du chapitre 22, ce qui semble bien être une réponse aux critiques de La Vallière. Le curieux effet de disjonction qui se trouve dans le manuscrit au moment où les deux versions du chapitre 22 se succèdent ne se comprend qu'avec l'hypothèse de deux envois séparés.

Les échanges épistolaires entre Voltaire et le duc de La Vallière qui nous sont parvenus commencent en 1755 (D6184) et continuent jusqu'en 1768 (D14698), et on aimerait en savoir plus sur leurs relations. Dans la première lettre qui nous est parvenue de La Vallière à Voltaire, il le remercie de lui avoir envoyé le Poème sur le désastre de Lisbonne (D6760), il lui envoie aussi La Pucelle, mais nous n'avons aucun des échanges qui eurent lieu au moment des envois de Candide. Seule trace, la lettre du duc datée du début 1759, tout de suite après la publication de Candide : «Le docteur Ralph s'est donc fait mettre sous la presse, et Candide a paru il y a huit jours. [...] L'on pense comme moi sur le chapitre de Paris, c'est le seul qui soit trouvé faible » (D8072). Cette référence au problématique chapitre 22 doit faire allusion aux échanges, aujourd'hui perdus, qui furent à l'origine de la réécriture et du deuxième envoi du chapitre. 
Voltaire n'a pas gardé systématiquement ses brouillons de travail. Le manuscrit de La Vallière qui éclaire partiellement la genèse de Candide est un document exceptionnel à plusieurs égards, mais sa survie n'est pas non plus un effet du hasard. Voltaire a rédigé Candide en gardant le secret absolu : nous ne trouvons aucune allusion à ce texte dans la correspondance. Dans le cas de La Vallière par contre, non seulement il lui demande son avis, mais il prend soin aussi de lui envoyer un manuscrit (plus ou moins) propre. Pour des raisons d'amitié, certes, et aussi parce qu'il appréciait ses conseils. Mais peut-être aussi pour des raisons stratégiques : en cas de censure, il aurait été utile d'avoir un manuscrit du roman logé chez un ami discret, sûr et bien placé. La survie de ce manuscrit remarquable n'a donc rien de fortuit. Il fut l'objet d'un don de la part de Voltaire, un don destiné à faire plaisir au destinataire certes, mais aussi un don qui faisait partie des stratégies de publication du roman.

Dans le cadre d'une étude génétique, l'objet de notre étude est par définition un manuscrit (des manuscrits) qui aboutit (aboutissent) à l'imprimé. Mais tous les manuscrits ne sont pas de ce genre, car Voltaire est aussi auteur de manuscrits destinés à rester tels. Voltaire trouve plaisir à faire don de manuscrits à ses amis. Impossible de ne pas penser d'abord à sa correspondance, inégalée au XVIII siècle tant pour le nombre de lettres que pour le nombre de correspondants : une lettre du grand homme devint tôt un objet prisé. Il offrait parfois des manuscrits plus substantiels, mais rarement, comme J.-J. Rousseau, des manuscrits d'apparat ; Voltaire préférait des manuscrits modestes et cultivait le don improvisé. Il envoie des vers à ses amis, et certains lui écrivent en vers pour l'obliger à composer des vers dans sa réponse ; une fillette de 11 ans qui lui écrivait à l'occasion de sa fête reçoit du patriarche, alors âgé de 81 ans, un petit poème en retour ${ }^{17}$. À Cideville, ami rouennais de longue date, Voltaire envoie en 1735 un « Recueil de poésies » : « Il y a si longtemps que je vous en promets une petite édition manuscritte que $\mathrm{j}$ 'aurois eu depuis le temps de composer un infolio » (D799, circa novembre 1734). Ce soi-disant « Recueil Cideville » est un manuscrit précieux, collection de poésies fugitives, dont beaucoup inédites à l'époque, parfois avec des variantes qu'on ne connaît pas par ailleurs ; nous trouvons même dans ce recueil un petit conte en vers, Le Janséniste et le moliniste, publié pour la première fois seulement au $\mathrm{xx}^{\mathrm{e}}$ siècle ${ }^{18}$. Dans le cas de Candide que nous avons déjà considéré, Voltaire envoie au duc de La Vallière un manuscrit pendant la période de gestation de l'œuvre : le manuscrit précède l'imprimé. Dans le cas du «Recueil Cideville », Voltaire crée une œuvre manuscrite permettant à son ami d'avoir accès aux poèmes qui n'étaient pas tous connus du grand public : le manuscrit prend la place de l'imprimé.

Voltaire fait don de manuscrits à ses amis, c'est clair, mais tous les manuscrits voltairiens ne sont pas destinés à un seul destinataire privilégié. Nous avons mis longtemps à comprendre que Voltaire est aussi un auteur de manuscrits, je veux dire par là qu'il se

17. Voir N. Cronk, « Voltaire et la réponse poétique », dans Séries et variations : études littéraires offertes à Sylvain Menant, dir. Luc Fraisse, Paris, PUPS, 2010, p. 771-784.

18. Voir E. Meyer, «Variantes aux Poésies mêlées de Voltaire d'après le manuscrit envoyé par l'auteur à M. de Cideville en $1735 », R H L F, \mathrm{n}^{\circ}$ 40, 1933, p. 400-423.

\section{Le manuscrit remplace l'imprimé : Voltaire auteur de manuscrits}


sert du manuscrit comme moyen de publication ${ }^{19}$. Prenons l'exemple de l'Épître à Uranie, expression précoce du déisme voltairien. Ce poème circule sous forme manuscrite dès 1722, mais ne sera imprimé avec l'autorisation de Voltaire que cinquante ans après, en 1772. Il n'est pas vrai, comme on le suggérait jadis, que Voltaire mit un demi-siècle à se décider à publier son poème... Lorsqu'il composa ce poème, dans les années 1720 , ce fut dans le but de le « publier » par voie manuscrite ; et lorsqu'il finit par l'imprimer dans les années 1770 , dans un climat politique et religieux très différent, le but polémique du poème n'était plus le même20.

Dans un premier temps, on choisit la voie manuscrite pour faire circuler un texte pour des raisons juridiques. Il est facile de renier un manuscrit. Et si jamais un éditeur se procure un exemplaire pour en faire une édition imprimée, Voltaire peut dire, l'air innocent, qu'il n'est vraiment pas au courant de l'édition. Le Sermon des cinquante, attaque cinglante contre le catholicisme, commence à circuler sous forme manuscrite à partir de $1752^{21}$. Voltaire parle d'" une espèce de sermon philosophique »- et l'attribue tout de suite à La Mettrie. Un manuscrit est dans la plupart des cas anonyme, et même si un nom d'auteur s'y attache, cette attribution n'a pas la même force juridique que dans le cas d'un imprimé. Lorsque J.-J. Rousseau, dans ses Lettres de la montagne (1764), fait allusion à Voltaire comme l'auteur du Sermon des cinquante, il lui porte un coup dur, même si ce qu'il révèle n'est qu'un secret de Polichinelle, car il ose dévoiler ce qui était censé rester non dit. Cramer finit par imprimer le texte en 1762, sous un pseudonyme bien sûr - mais même dix ans après, l'édition est antidatée à 1749 , précaution de plus pour semer de la confusion chez les censeurs...

Un texte comme le Sermon des cinquante s'inscrit clairement dans la tradition du manuscrit clandestin, mais comme nous le rappelle François Moureau, il est erroné de présumer que toute publication manuscrite est forcément philosophique et clandestine 22 . Comme nous l'avons vu avec l'Épître à Uranie, Voltaire compose aussi des poèmes manuscrits, dont le plus célèbre est La Pucelle. Les nombreux manuscrits de ce poème se divisent en deux groupes distincts : ceux qui précèdent la première publication du poème, et ceux qui sont postérieurs à l'édition Cramer de 176223. En 1755, à un moment où les éditions non autorisées se multiplient, Voltaire réagit en envoyant à de nombreux amis un manuscrit : la publication manuscrite, il faut l'appeler ainsi, de La Pucelle est ici une réponse à la publication des versions imprimées non autorisées. Le manuscrit voltairien n'est pas toujours destiné à devenir un imprimé, car Voltaire est aussi un auteur d'œuvres manuscrites.

19. Voir La Lettre clandestine, $\mathrm{n}^{\circ} 16,2008$, «Voltaire et les manuscrits philosophiques clandestins », et Revue Voltaire, $n^{\circ} 8,2008$, «Approches voltairiennes des manuscrits clandestins ». Voir aussi Miguel Benítez, « Voltaire and clandestine manuscripts », dans The Cambridge Companion to Voltaire, dir. N. Cronk, Cambridge, CUP, 2009, p. 65-77.

20. Voir N. Cronk, «L'Épître à Uranie de Voltaire : stratégies de publication d'une œuvre clandestine », dans B. Guion et al. (dir.), Poétique de la pensée : études sur l'Âge classique et le siècle philosophique : en hommage à Jean Dagen, Paris, Champion, 2006, p. 275-284.

21. Voir l'édition critique de J. Patrick Lee et Gillian Pink, $O C V$, t. XLIXA, 2010.

22. La Plume et le plomb, op. cit., p. 12-20.

23. Voir l'édition de J. Vercruysse, OCV, t. VII, 1970 ; et N. Cronk, « Two manuscripts of La Pucelle in the New York Public Library », Revue Voltaire, $n^{\circ}$ 9, 2009, p. 127-129. 
Le troisième cas de figure qui se présente est celui où le manuscrit vient après l'imprimé, en ajout en quelque sorte. Le phénomène du mélange de l'imprimé et du manuscrit mérite d'être davantage étudié. Dans la culture des colonies américaines par exemple, au début du xvIII siècle, un objet très répandu était le formulaire juridique : pour toutes sortes de transactions, on achetait un formulaire imprimé, et ensuite on insérait à la main un nom, une somme, etc. : "There was only one kind of printed artifact that could have been regarded as an everyday secular object, a piece of the standard routine of early-eighteenth-century society. That was the legal form ${ }^{24}$. » De même nous trouvons des livres imprimés portant des traces manuscrites, et nous avons affaire ici à un phénomène caractéristique de la pratique voltairienne.

Considérons d'abord l'exemple de Mahomet (1742), tragédie dont on connaît une dizaine de manuscrits 25 . Ils ont tous un statut un peu différent et sont de valeur inégale : il y a un manuscrit qui remonte à la première composition de la pièce, où des vers ont été remplacés, ou déplacés, et une partie du texte a été amplifiée ; il y a des copies contemporaines et des copies ayant appartenu à des acteurs ; il y a même un manuscrit qui a appartenu à Le Kain, acteur qui créa le rôle de Mahomet. Mais le statut même de ce que l'on peut appeler «manuscrit » est parfois complexe, comme le suggère l'édition imprimée de Mahomet qui se trouve dans les collections manuscrites de la $\mathrm{BnF}^{26}$. Le « manuscrit» en question figure dans la collection de Cayrol, dans un volume de correspondance entre Voltaire et Missy : ce dernier était chapelain de l'église française de Saint-James à Londres de 1741 à 1745, et aida Voltaire à plusieurs reprises. Après la création de Mahomet à Paris en août 1742, Voltaire décida de publier sa pièce, et sa première idée était de faire appel à César de Missy pour l'aider à l'éditer à Londres 27 . Une première édition pirate parut rapidement à Meaux ${ }^{28}$; et c'est à partir de cette édition que Voltaire semble avoir préparé le texte « définitif » de sa tragédie. Il envoya à Missy un exemplaire de l'édition imprimée, richement annoté, et c'est cet objet qui se trouve toujours dans la collection de Cayrol29. L'édition «approuvée » de Londres n'a jamais vu le jour, et la première édition pirate fut suivie par d'autres qui parurent à Paris avec pour lieu d'impression « Bruxelles »; Voltaire laissa tomber l'idée d'une édition londonienne.

Ce manuscrit de la BnF est donc un objet curieux : le témoignage d'un projet d'édition avorté. Sur la page de titre se lisent deux tampons : en bleu, « Ex libris de Cayrol », et en rouge, «Bibliothèque impériale MSS », ce qui confirme son acquisition par la Bibliothèque sous le Second Empire. La main de Voltaire s'y trouve aussi : après « Mahomet », il

24. Michael Warner, The Letters of the Republic: Publication and the Public Sphere in Eighteenth-Century America, Cambridge, Mass., Harvard University Press, 1990, p. 18.

25. Voir l'édition critique de C. Todd, $O C V$, t. XXв, 2002, p. 49-60.

26. BnF, Ms. fr. 12933, fos 74-123. Je remercie chaleureusement Michèle Sacquin, conservatrice en chef au département des Manuscrits de la $\mathrm{BnF}$, qui a attiré mon attention sur ce manuscrit et a beaucoup facilité mes recherches au département des Manuscrits.

27. Voir les lettres que Voltaire adresse à Missy entre septembre et décembre 1742 : D2648, D2676, D2682, D2684 et D2699.

28. Voir Le Fanatisme, ou Mahomet le prophète, éd. C. Todd, $O C V$, t. XXB, p. 60-64, édition « 42XI »; sur les échanges avec Missy, voir p. 32-33 ; et D2660 et D2661.

29. Voir l'édition $O C V$, p. 64-65. Cette version manuscrite y est décrite parmi les éditions imprimées sous le sigle « $42 \mathrm{XI}^{*} »$.
Le manuscrit succède à l'imprimé : les imprimés portant des touches manuscrites 
ajoute « ou le fanatisme » (fig. 1), soulignant ainsi la portée philosophique de ce poème théâtral ${ }^{30}$. L'édition imprimée a été démontée complètement, et on peut penser que c'était peut-être déjà sous cette forme que Voltaire l'envoya à Missy : dans ce cas, il aurait luimême transformé le statut de l'imprimé en manuscrit. Sur les pages imprimées nous trouvons deux couches manuscrites, de couleur différente, qu'il importe de distinguer ${ }^{31}$. Premièrement, à l'encre noire, les ajouts autographes de Voltaire. Et en second lieu, une série de marques, accompagnées de quelques notes, qui sont en rouge, et qui sont d'une autre main. Je traiterai ces deux couches d'annotations successivement.

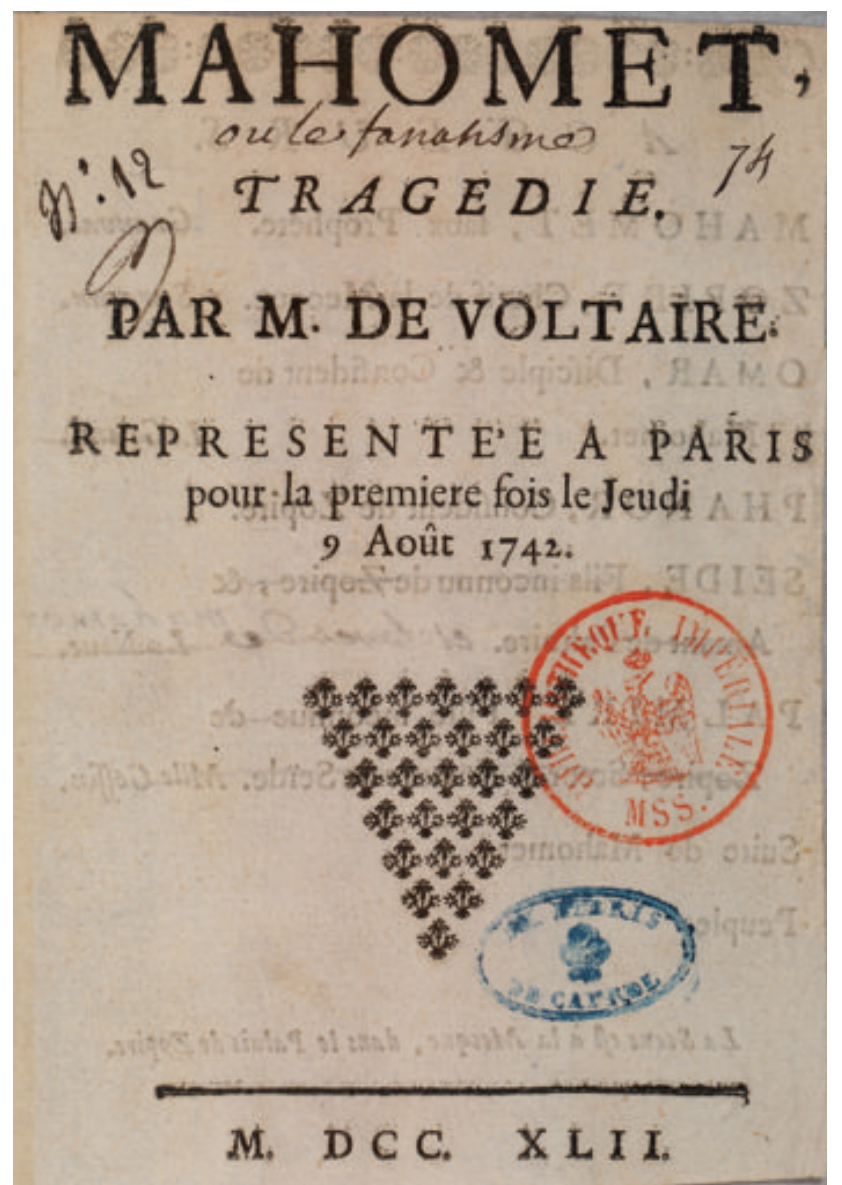

Fig. 1 : Voltaire, Mahomet, Page de titre (BnF, Ms. fr. 12933, $\left.\mathrm{f}^{\circ} 74 \mathrm{r}^{\circ}\right)$

30. Voltaire ajoute après « Mahomet » les mêmes mots « ou le fanatisme » à la page 1, dans le titre de départ. 31. Dans la description qui se trouve dans l'édition $O C V$ (p. 64-65), on ne distingue pas entre ces deux couches : la distinction ne se voit nullement d'ailleurs sur le microfilm qu'on consulte habituellement. Dans le cas actuel, il est indispensable pour le chercheur de pouvoir consulter l'objet original pour bien comprendre la complexité de sa genèse. 


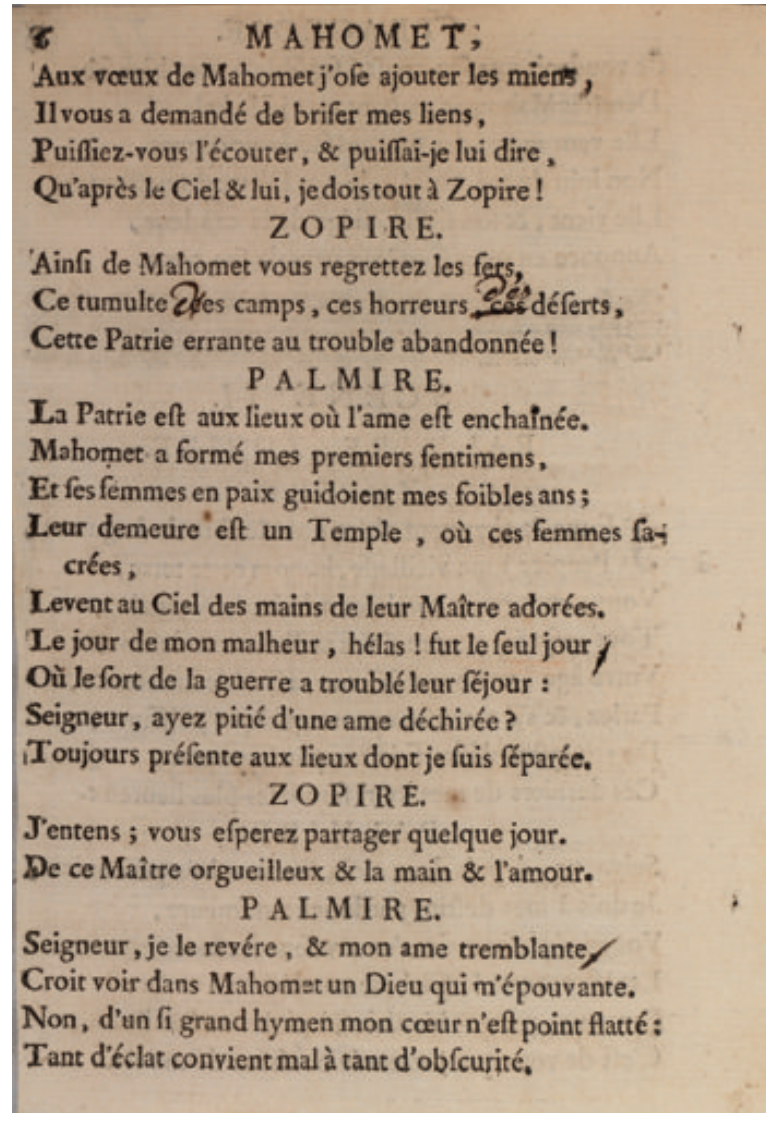

Fig. 2 : Voltaire, Mahomet (BnF, Ms. fr. 12933, fo $77 \mathrm{v}^{\circ}$, p. 6)

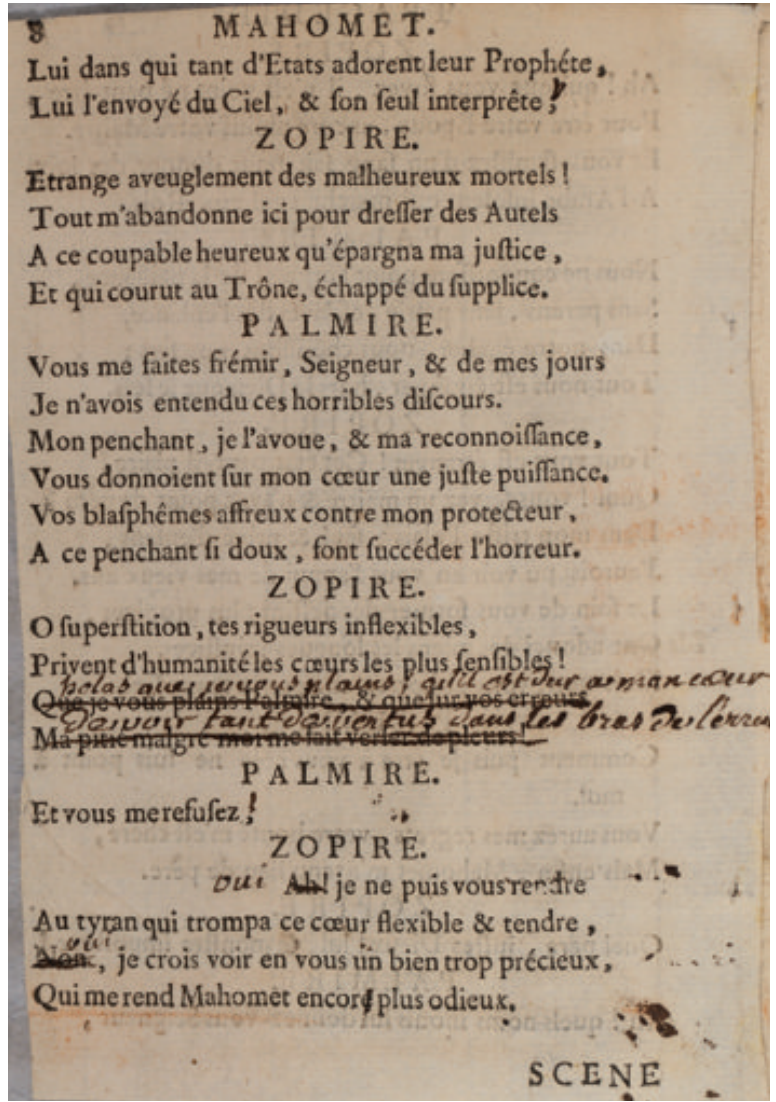

Fig. 3 : Voltaire, Mahomet (BnF, Ms. fr. 12933, fo $78 \mathrm{v}^{\circ}$, p. 8)

Les corrections autographes de Voltaire sont toutes en noir et elles sont de plusieurs types. Il y a d'abord des corrections modestes et habituelles : Voltaire corrige la ponctuation, par exemple, ou bien il change « ces » en « des », et vice versa (fig. 2). Ensuite, il y a les cas plus importants lorsque Voltaire modifie et améliore son texte. Il ajoute, par exemple, un vers qui manque 32 ; il réécrit des vers qui existent déjà (fig. 3$)^{33}$; et à un autre moment, deux vers sont carrément rayés ${ }^{34}$. Lorsqu'il y a des ajouts ou des réécritures très importants, Voltaire écrit le texte sur un bout de papier qu'il colle dans l'imprimé, en cachant le texte qu'il veut supprimer : c'est ainsi que le manuscrit vient s'ajouter à l'imprimé et parfois même le supprimer. Par exemple, à la page 13 (acte I, scène IV), un ajout est collé en bas de page, et un texte de six vers remplace les deux vers

32. P. 2 ; acte I, scène I (OCV , p. 167, vers 11). Pour des raisons de simplicité, mes références au manuscrit de la BnF sont aux numéros de page de l'imprimé et non pas aux numéros de folios.

33. P. 8 ; acte I, scène II $(O C V$, p. 178, variantes aux vers 159-160).

34. P. 87 ; acte V, scène IV (OCV, p. 297, variantes aux vers 154-156, et note 13). 
de l'imprimé. Omar, le lieutenant de Mahomet, essaie ici de convaincre Zopire de servir Mahomet ; dans la première édition de 1742, Voltaire avait écrit :

Ouvre les yeux, Zopire, et change ainsi que moi :

Reconnais une loi qui s'étend par la guerre,

Tu me vois après lui le premier de la terre [...].

Maintenant pour l'édition révisée Voltaire étend ce discours :

Ouvre les yeux, Zopire, et change ainsi que moi :

Et loin de me vanter les fureurs de ton zèle,

Ta persécution, si vaine et si cruelle,

Nos frères gémissants, notre dieu blasphémé,

Tombe aux pieds du héros par toi-même opprimé.

Viens baiser cette main qui porte le tonnerre.

Tu me vois après lui le premier de la terre ${ }^{35}$.

En allongeant le discours d'Omar, Voltaire affine la psychologie de son appel : la bonne foi de Zopire est transformée en "zèle », tandis que Mahomet devient plus qu'un conquérant, il est un «héros ». Même chose à la page 59 (acte IV, scène I), dans un discours de Mahomet, où dix vers remplacent un passage beaucoup plus court dans l'original ${ }^{36}$. À certains moments, le manuscrit collé recouvre presque la page entière ${ }^{37}$, voire la page entière, comme c'est le cas pour le début du troisième acte (fig. 4). La fin de ce troisième acte est de même réécrite entièrement sur deux feuilles collées ${ }^{38}$.

À côté de, et en parallèle avec, ces révisions parfois importantes du texte, nous sommes confrontés également aux marques en rouge qui témoignent d'une autre lecture du texte ; et on trouve même des interventions en rouge sur les pages purement manuscrites, ce qui confirme qu'il s'agit d'une deuxième main et d'une lecture postérieure (fig. 4). Des traits (parfois des doubles traits) en marge renvoient à des fautes d'impression : lorsque l'imprimé propose «emnemi » (p. 17), la faute est soulignée en rouge, et un double trait en marge attire notre attention sur la correction à faire ; même chose pour « snblime » (p. 23), qui est souligné en rouge avec un trait en marge. À certains moments, ce relecteur apporte en rouge des traits censés attirer l'attention sur les corrections autographes de Voltaire, lorsqu'il élimine une virgule, par exemple, ou quand il insère un « et » (fig. 5). Sur cette même page 20, nous voyons une croix rouge, et en avançant dans le manuscrit, les croix remplacent de plus en plus les traits. Parfois le travail de ce relecteur va plus loin, et il lui arrive de faire des notes en marge, avec des renvois dans le texte : c'est ainsi que "Moad» est corrigé, avec un renvoi à une autre page où effectivement nous lisons « Morad » (fig. 6) ${ }^{39}$. Tout ceci fait partie de la préparation du texte pour la presse : on attire l'attention du prote sur les corrections de Voltaire, et on note d'autres détails qui lui ont échappé.

35. Voir l'édition $O C V$, p. 186-187 (vers 261-266 et variantes).

36. Voir l'édition $O C V$, p. 253-254 (vers 19-28).

37. C'est le cas à la page 43 (acte III, scène III) ; OCV, p. 228-231.

38. P. 54-55; $O C V$, p. 246-250.

39. Voir aussi D2689 : Missy hésite sur la bonne lecture ici. 


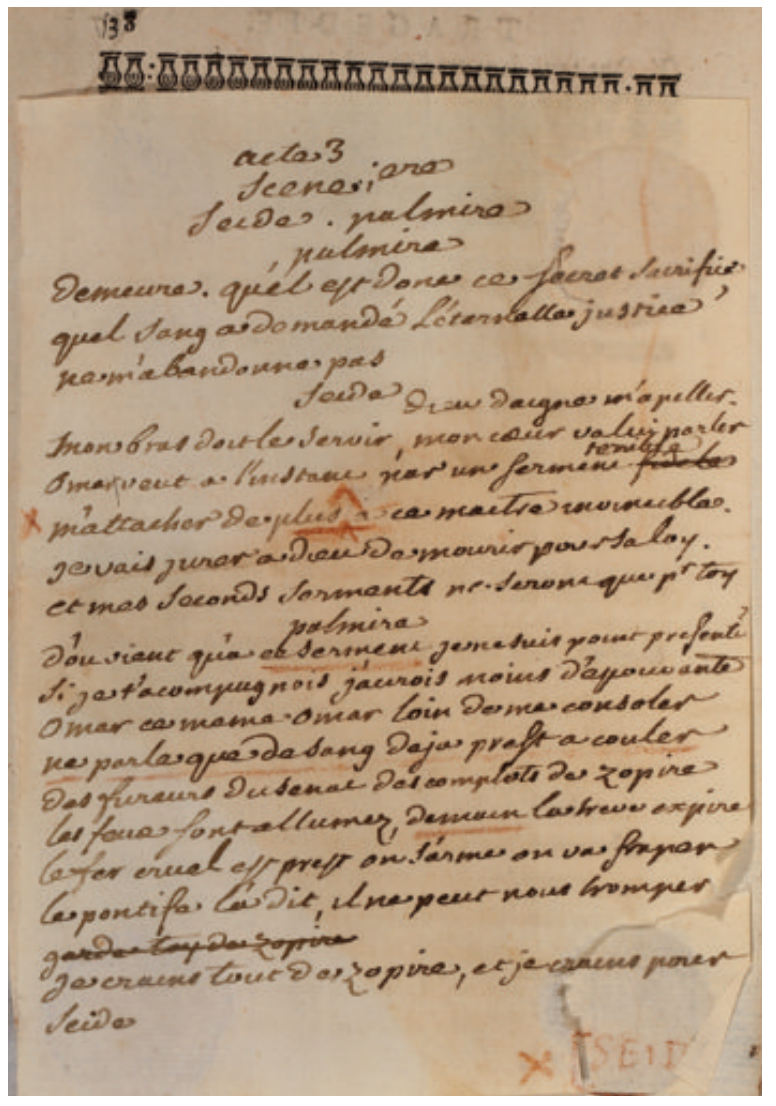

Fig. 4 : Voltaire, Mahomet (BnF, Ms. fr. 12933, fo $93 \mathrm{v}^{\circ}$, p. 38)
20. M A H O E T,

D'aucune ombre d'efpoir n'étoit plus fecoura;

Tout fioiffoit pour moi, quand Seide a paru. SE I D E.

Quel eft donc ce mortel inflexible à tes larmes? P A L. M I R E.

C'eft Zopire; il fembleit touché de mes allarmes;

Mais le cruel enfin vient de me déclarer

Que des lieux où je fuis rien ne me peut tirer.

S E I D E.

Le barbare fe trompe; \& Mahomet mon maître,

Et l'invincible Omar, \&ton Amant peut-être,

(Car j’ofe me nommer après ces noms fameux, Pardonne a ton Amant cet efpoir orgueilleux )

Nous briferons ta chaîne, \& tarirons tes larmes ;

Le Dieu de Mahomet protecteur de nos armes,

Le Dieu dont j’ai porté les facrés étendarts,

Ce Dieu qui de Medine a détruit les ramparts,

Renverfera la Mecque à nos pieds abbatue?

Omar eft dans la Ville, \& le peuple à fa vûe

N'a point fait éclater ce trouble, cette horreur

Qu'infpire aux ennemis un ennemi vainqueur :

Au nom de Mahomet un grand deffein l'amene.

\section{P A L M I R E.}

Mahomet nous chériț il briferoit ma chaine !

Il uniroit nos cceurs! Nos cceurs lui font offerts :

Mạs il eft loin de nous, \& nous fommes aux fers,

Fig. 5 : Voltaire, Mahomet (BnF, Ms. fr. 12933, fo $84 v^{\circ}$, p. 20$)$

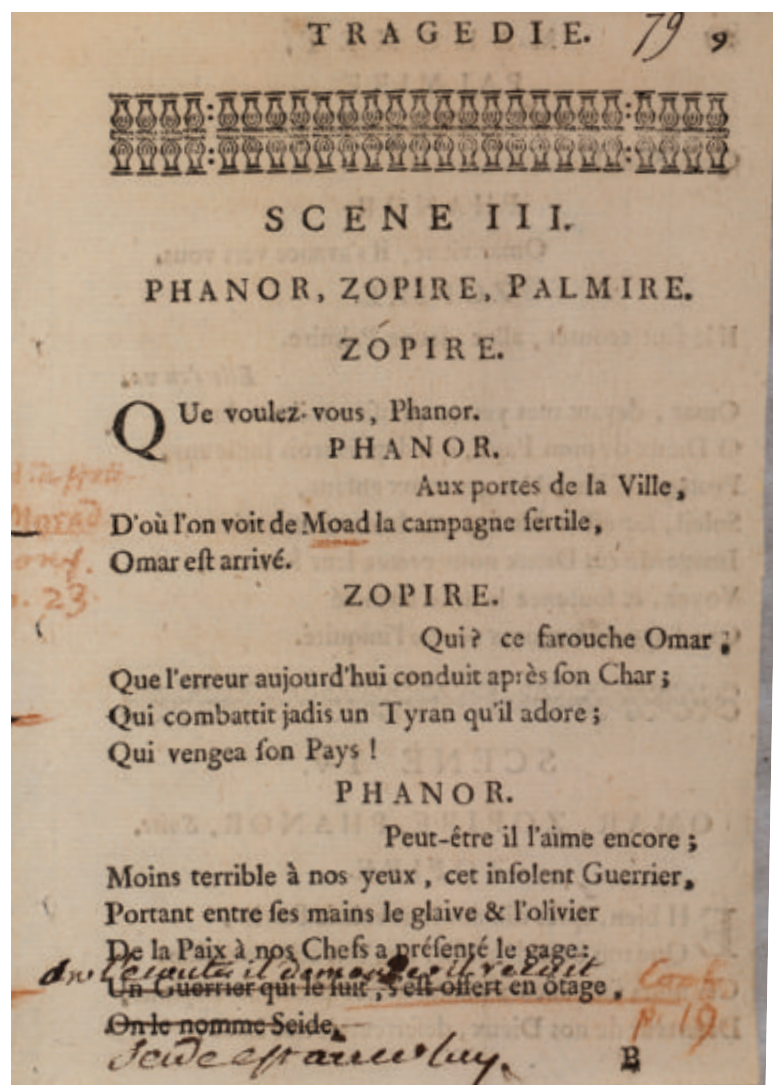

Fig. 6 : Voltaire, Mahomet (BnF, Ms. fr. 12933, $\mathrm{f}^{\circ} 79 \mathrm{r}^{\circ}$, p. 9) 
D'autres marques en rouge sont plus ambitieuses, et parfois elles désignent un passage où il n'y a aucune faute d'impression. Par exemple, on souligne en rouge l'expression «nos cœurs lui sont offerts » (fig. 5) : le texte à cet endroit n'a jamais changé 40 , et on ne peut que supposer que le lecteur au crayon rouge n'a pas trop apprécié cette répétition de «cœurs » qui figure deux fois dans le même vers. De même, à la page 19 (acte II, scène I), un trait vertical désigne un passage du texte qui ne semble pas fautif ${ }^{41}$. Sur la page 51 (acte III, scène VIII ; fig. 7), nous retrouvons toute une gamme de corrections. Le relecteur au crayon rouge met une croix en marge pour noter une faute d'orthographe (" tu t'étonne »), et il change un point-virgule en point d'exclamation. Tout cela est clair. Mais pourquoi met-il deux parenthèses avec deux croix pour marquer un discours de cinq vers prononcé par Séïde 42 ? Il n'y a aucune faute de typographie à corriger ici, et il faut conclure que c'est plutôt la qualité des vers euxmêmes qui est mise en question. Ce n'est pas un ouvrier de la presse qui se serait permis de telles interventions sur un texte de Voltaire (de toute façon le manuscrit n'arriva peut-être jamais chez l'imprimeur londonien), et on commence à deviner la présence de Missy lui-même dans ce commentaire en rouge. L'identité du relecteur semble confirmée à la page précédente, où dans un discours de Zopire le mot « cœur » est souligné en rouge (fig. 8) : nous savons par une lettre qu'il adressa à Voltaire que Missy préféra ici le mot « œil », et que Voltaire finit par adopter cette suggestion ${ }^{43}$. Il serait difficile de confirmer l'écriture de Missy à partir des quelques exemples qu'offre ce manuscrit ${ }^{44}$, mais le contexte de l'envoi comme le contenu du manuscrit semblent bien suggérer que la deuxième couche rouge du commentaire vient de cet interlocuteur de Voltaire. Nous connaissons l'exemple d'un livre annoté de la main de Voltaire où l'encre se présente en deux couleurs distinctes, et dans ce cas, les deux couleurs témoignent de lectures faites à des moments distincts, mais toujours par la même personne ${ }^{45}$. Dans le cas du manuscrit actuel, les deux couleurs (et les deux styles d'annotation) témoignent de deux lecteurs distincts.

Ce manuscrit de Mahomet est un objet fascinant, mais aussi un objet difficile à interpréter. Il témoigne de l'existence d'une édition londonienne qui n'a jamais paru, et contribue donc à la genèse virtuelle d'un texte qui évolua beaucoup. En fait, la plupart des corrections et des ajouts apportés par Voltaire sont entrés dans d'autres éditions par la suite et continuent à figurer dans l'édition « définitive » qui finit par s'établir - ce qui prouve que Voltaire a dû garder une copie des corrections qu'il envoya à Missy. Restent les quelques cas où ce manuscrit présente des variantes manuscrites autographes qui

40. Voir l'édition $O C V$, p. 196, vers 55.

41. Voir l'édition $O C V$, p. 194, vers 21-24. Encore une fois, il s'agit d'un passage qui est resté stable dans les éditions ultérieures.

42. $O C V$, p. 242-243, vers 228-232.

43. Voir l'édition $O C V$, p. 242, vers 218 , et variante ; voir aussi D2689.

44. Ce manuscrit se trouve dans un volume qui contient des lettres autographes de Missy : l'écriture n'est certes pas très différente, même s'il serait difficile d'affirmer qu'il s'agit de la même main.

45. N. Cronk, «Voltaire (non) lecteur de Nieuwentijt : le problème des causes finales dans la pensée voltairienne », Revue Voltaire, ${ }^{\circ} 7,2007$, p. 169-181 ; voir p. 179-180, et planches III et IV. 


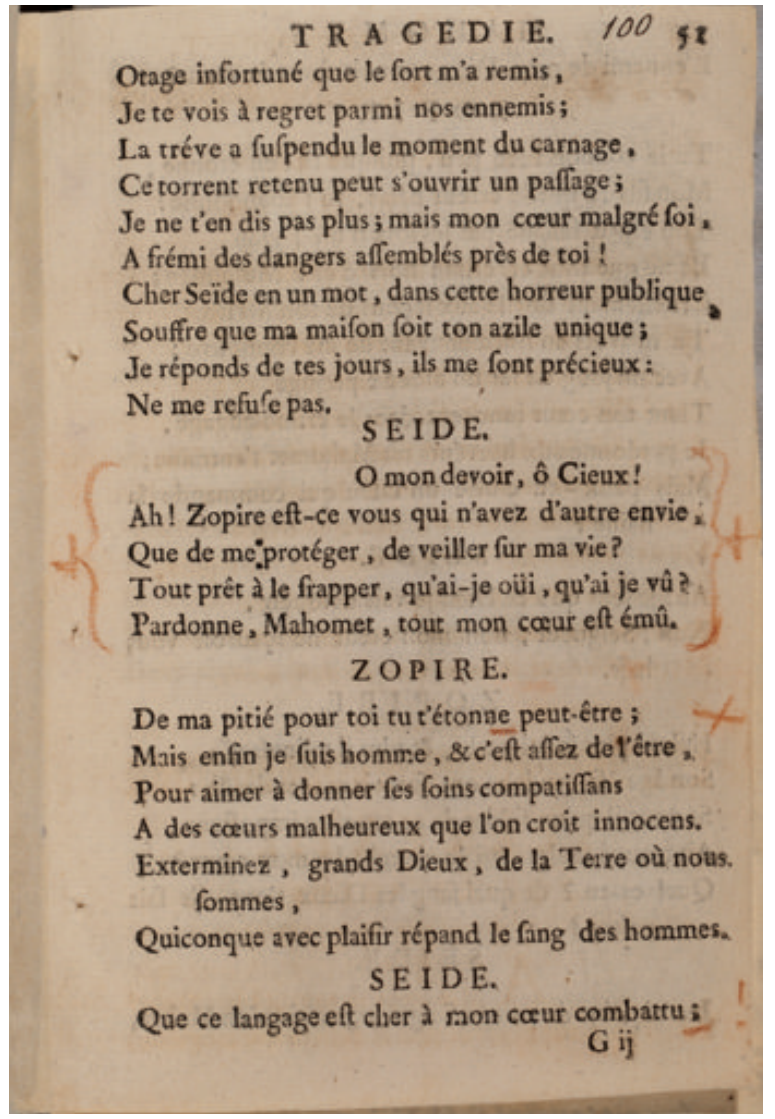

Fig. 7 : Voltaire, Mahomet (BnF, Ms. fr. 12933, fo 100 rº p. 51)

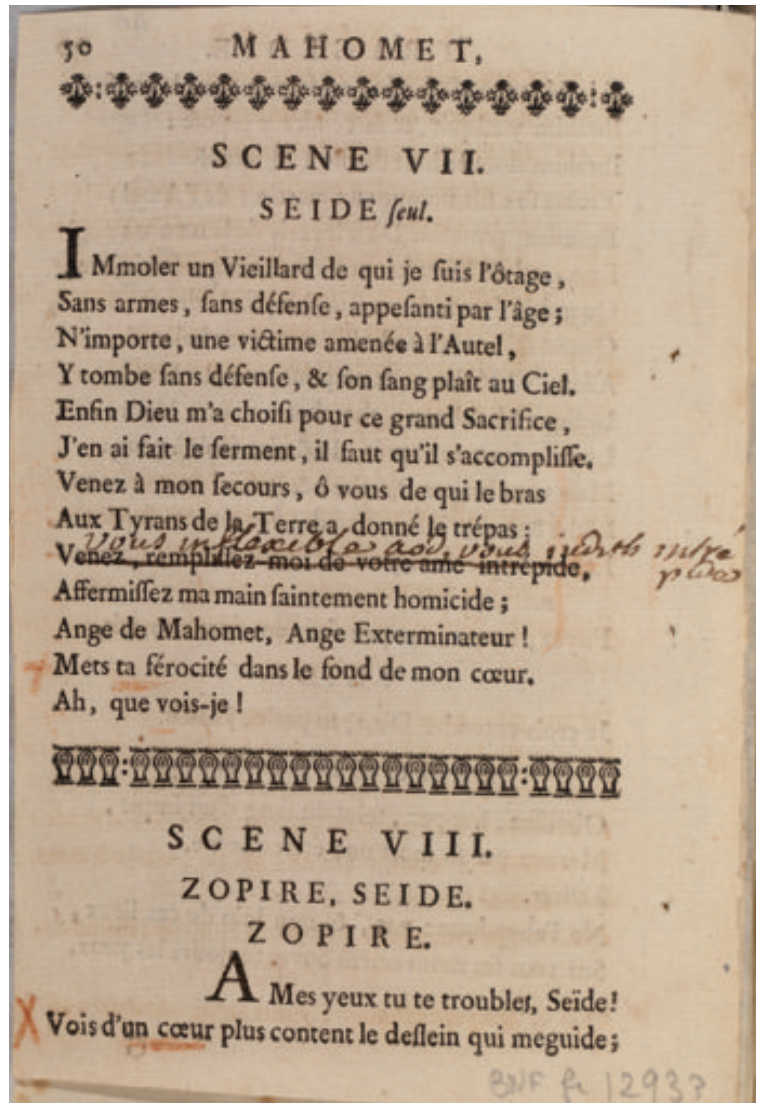

Fig. 8 : Voltaire, Mahomet (BnF, Ms. fr. $12933, f^{\circ} 99 v^{\circ}$, p. 50)

n'existent nulle part ailleurs et qui figurent seulement dans ce manuscrit : c'est le cas pour les deux vers qu'il réécrit dans un discours de Zopire dans la deuxième scène du premier acte (fig. 3) ${ }^{46}$; c'est aussi le cas pour un vers dans un discours de Séïde (acte III, scène VII ; fig. 8$)^{47}$. À ces quelques exceptions près, ce manuscrit ne change pas de façon significative nos connaissances concernant la genèse du texte, et son vrai intérêt se trouve peut-être ailleurs. Témoin d'un projet d'édition avorté, ce manuscrit est aussi le témoin d'une façon de travailler, et surtout d'une façon de collaborer. Loin d'être un objet inerte, ce manuscrit en deux couleurs et à deux voix a été un outil de communication entre Voltaire et Missy, un lieu de questionnements plutôt que d'affirmations. Il nous présente un texte qui bouge en permanence, un texte qui se met perpétuellement en question. Le mélange assez particulier d'imprimé et de manuscrit ne fait que souligner le caractère dynamique de l'objet.

Le «manuscrit» de Mahomet est un objet de travail. Dans d'autres cas, un imprimé portant des traces d'écriture fait l'objet d'un don. Dans le cas d'un livre dédicacé, la pratique est habituelle, mais Voltaire va souvent plus loin, cédant à la tentation de corriger, voire d'ajouter. Une collection « complète » des œuvres de Voltaire parait en quatre tomes chez Ledet à Amsterdam en 1738-1739. Il s'agit d'une édition qui fait date dans la constitution du canon voltairien, mais Voltaire était très déçu par la présence dans

46. Voir l'édition $O C V$, p. 178, variantes aux vers 159-160.

47. Voir l'édition $O C V$, p. 241, variante au vers 213. Sur toutes les variantes propres à ce manuscrit, voir p. 64 . 
l'édition d'un certain nombre de fautes typographiques ${ }^{48}$. De Bruxelles, où il était retenu pour une affaire judiciaire concernant $\mathrm{M}^{\mathrm{me}}$ Du Châtelet, Voltaire écrivit au marquis d'Argenson :

Mes petits hommages que je vous dois monsieur depuis longtemps sont partis par le coche $[\ldots]$; ce sont quatre volumes de mes réveries imprimées à Amsterdam. Les fautes des éditeurs se trouvoient en fort grand nombre avec les miennes. J'ay corrigé tout ce que j'ai pû, et il s'en faut baucoup que je n'en aye corrigé assez (D2210, 21 mai 1740).

C'est ainsi que Voltaire annonce en même temps l'envoi de l'édition en quatre tomes et le fait que cette édition est «personnalisée » par la présence de corrections autographes. Il envoie d'autres exemplaires corrigés au président Hénault, à Helvétius, à Cideville, à Thiriot. Il avertit Cideville : "Vous devez trouver dans cette édition beaucoup de corrections à la main, $[\ldots]$ mais surtout les fautes énormes de l'éditeur réformées tant que je l'ai pu » (D2251, 28 juin 1740) ; et à Thiriot : «Je vous enverrai mon édition, moitié imprimée, moitié manuscrite »(D2445, 13 mars 1741)49.

J'ai étudié, à titre d'exemple, cinq exemplaires de cette édition, corrigés tous apparemment de la main de Voltaire 50 . L'exemplaire (1) fut envoyé au président Hénault et contient son ex-libris, l'exemplaire (4) est celui qui fut envoyé à d'Argenson. Pour les trois autres éditions, on ne connaît pas l'identité du destinataire. La nature et le nombre des corrections varient considérablement entre les cinq éditions. L'exemplaire (5) est légèrement corrigé, l'exemplaire (2) aussi, avec des corrections au crayon qui semblent hâtives. Les exemplaires (1) et (4) sont plus lourdement corrigés, mais à l'encre et dans une écriture soignée : nous avons affaire ici à des exemplaires « de présentation » destinés à des amis, et à des amis qui bénéficiaient d'un certain statut social.

Parfois, il s'agit effectivement de corriger des bourdes de l'imprimeur : dans les exemplaires (1) et (4), mais non pas dans les trois autres, « le Maréchal de Morillac » est corrigé en «Marillac » (t. IV, p. 157). La phrase qui commence « Notre Descartes, né non pour découvrir les erreurs de l'Antiquité... » (t. IV, p. 235) est évidemment fausse : le «non » est barré dans les exemplaires (1), (3) et (4). À d'autres moments, Voltaire saisit l'occasion pour corriger sa propre pensée : en parlant des tourbillons cartésiens, il avait écrit : « tout solide, mu dans un fluide aussi dense que lui, perd toute sa vitesse »; dans les trois exemplaires (1), (3) et (4), Voltaire corrige en «perd la moitié de sa vitesse » (t. IV, p. 255). Et dans certains cas, Voltaire va plus loin, saisissant l'occasion pour faire des ajouts au texte. La sixième des Lettres philosophiques conclut avec une formule frappante devenue célèbre : "S'il n'y avait en Angleterre qu'une Religion, le Despotisme serait à craindre... ». Dans les deux exemplaires (1) et (4), destinés à Hénault et à d'Argenson, Voltaire introduit une variante manuscrite importante : « S'il n'y avoit en Angleterre et en

\footnotetext{
48. Voir l'édition critique par N. Cronk du Mémoire sur l'édition des æuvves de Monsieur de Voltaire, faite à Amsterdam chez Desbordes et Ledet, 1739 (OCV, t. XVIIIв, 2007, p. 411-429).

49. Et encore à Thiriot, «Je vous enverray cette édition moitié imprimée moitié manuscritte, vous y trouverez quelques changements à la Henriade, et à tous mes autres ouvrages »(D2457, 6 avril 1741).

50. Je me réfère aux cinq exemplaires suivants : (1) Paris, BnF, Z Beuchot 4 ; (2) Paris, BnF, Z Bengesco 468 ; (3) Paris, Arsenal, Rés. 8 o BL 3042 (1-4) ; (4) Paris, Arsenal, Rés. 8 o BL 3043 (1-4) ; et (5) Bruxelles, Bibliothèque royale, FS $277 \mathrm{~A}$.
} 
Hollande qu'une Religion, le Despotisme serait à craindre... » (t. IV, p. 194 ; c'est moi qui souligne). Cette dernière variante, fascinante pour notre lecture des Lettres soi-disant anglaises, ne se trouve dans aucune édition imprimée : il s'agit d'un «don » à deux lecteurs privilégiés.

Voltaire enrichit ainsi ses propres imprimés avec des ajouts manuscrits, et il lui arrive même d' 'enrichir » les imprimés des autres... On sait que Voltaire a beaucoup « marginé » les nombreux livres de sa bibliothèque - c'est lui d'ailleurs qui a forgé le néologisme « marginer»; en outre, dans certains cas Voltaire a marginé le livre d'un autre auteur dans le but d'en faire cadeau à un ami51. C'est ainsi qu'il offre à un voisin, David-Louis Constant de Rebecque, seigneur d'Hermenches, un exemplaire de l'Émile de Rousseau52. Le commentaire n'est guère appréciatif, et nous lisons en marge : "faux », « obscur et faux », «pitoyable », « lisez Loke »; plus rarement nous trouvons « bon », voire " très bon ». Il y a des questions rhétoriques : «pourquoi calomnier les philosophes ? », ou bien «pourquoi professer des sottises; il n'y a qu'à se taire, et ne rien professer ». Voltaire s'amuse à indiquer des sources non nommées : «imité des Pensées de Pascal », et mieux encore lorsque c'est lui qui est plagié, « tout ce discours se trouve notamment dans le poème de la religion naturelle et dans l'epitre à Uranie ». Voltaire entre en dialogue ainsi avec Jean-Jacques (« eh pauvre homme, n'est-ce pas là ton caractère ? »), et fait cadeau de cette saynète à son ami. Dans tous ces exemples, les touches manuscrites donnent une apparence improvisée, une touche d'authenticité aussi, elles constituent une sorte de signature. Et elles nous rappellent que l'écriture chez Voltaire (comme d'ailleurs sa signature...) est toujours en mouvement.

Nous avons tendance à imaginer Voltaire comme un homme du livre plutôt que du manuscrit, et c'est faux. De Bruxelles, il écrit à Frédéric II, « je suis ici sans mes manuscrits et sans mes livres » (D2125, 28 décembre 1739) : il a besoin des deux pour travailler. Wallace Kirsop parle d'une «cohabitation équivoque » entre les manuscrits et les imprimés au siècle des Lumières ${ }^{53}$, et dans cette perspective, Voltaire se trouve à cheval

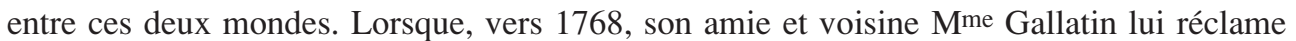
un exemplaire de son dernier brûlot, Voltaire lui répond : "Madame, Étant privé depuis huit jours d'un de mes copistes, je n'ai pu encor vous obéir. On me dit dans le moment que cette sotise court imprimée à Genève. J'en doute fort. Je m'en informerai, et vous l'aurez soit imprimée, soit manuscrite » (D15115). Le manuscrit est parfois donc un simple substitut pour le livre.

Les manuscrits voltairiens impressionnent. Voltaire fait don d'un exemplaire de ses œuvres à Elisabeth, princesse de Anhalt-Zerbst (mère de la future Catherine II) ; dans son accusé de réception, cette dernière fait allusion à « la belle inscription » qu'il a eu « la politesse d'y ajouter », et elle continue :

Mais vous n'avez peut-être pas senti, Monsieur, ce que vous m'allez imposer par là ; vous me mettez dans l'obligation de former une Bibliothèque pour soutenir la réputation de femme lettrée que vôtre

51. Voir N. Cronk, «Les notes marginales de Voltaire : quel est le lectorat visé ? », OCV, vol. CXXXIX, p. 763-779. 52. Voir $O C V$, t. CXLIII, Appendice.

53. Cité par F. Moureau, La Plume et le plomb, op. cit., p. 11. 
présent me done. Il y attirrera les Savens et les Personnes de goût pour consulter ce rare exemplaire de vos œuvres avec la même ardeur qu'on examine un manuscrit de Virgile ou de Ciceron (D4477, 25 mai 1751).

Le manuscrit voltairien est ainsi en train d'acquérir très tôt un statut de «classique ». Il y a eu un moment où les manuscrits de Voltaire ont fait peur aussi. En particulier, ils ont fait peur à Louis XVI. En 1774, le roi donna des ordres secrets pour mettre les scellés sur les papiers « dangereux » de Voltaire au moment même de sa mort. Ordre qui gêna beaucoup Pierre Michel Hennin, Résident de France à Genève depuis 1765, et ami de Voltaire. Hennin, qui risquait d'être chargé de l'exécution de ces ordres, réagit avec une précaution diplomatique ; les ordres furent suspendus, puis réactivés en 1777, mais l'affaire n'eut plus de suite 54 .

En réalité, ce ne sont pas les manuscrits en tant qu'objets qui auraient dû faire peur à Louis XVI. Ce qui surprend et impressionne chez Voltaire, c'est le manuscrit comme activité (il aurait pu créer un néologisme " manuscrire », comme il a créé " marginer »). Voltaire ne cesse d'écrire, et il se fait réécrire en permanence, souvent en se plaignant de ses copistes ${ }^{55}$. Il aime offrir le manuscrit, son manuscrit. La touche manuscrite a une importance plus que symbolique : on peut penser à la façon dont Voltaire ajoute son nom, de sa propre main, aux lettres rédigées par un secrétaire. Et plus que cela, le manuscrit garde un aspect spontané, la trace d'une écriture qui bouge. Voltaire possède, dans tous les sens, le don du manuscrit.

\footnotetext{
54. Voir D19164.

55. Voir D799 et D6364.
} 
Nicholas Cronk est professeur de littérature française à l'université d'Oxford, et directeur de la Voltaire Foundation. Il dirige l'édition des Euvres complètes de Voltaire qui est publiée à Oxford, et il est président de la Société des études voltairiennes. Il est l'auteur de The Classical Sublime: French Neoclassicism and the Language of Literature (2002) et il a édité de nombreux volumes, dont Les Notes de Voltaire : une écriture polyphonique (avec Christiane Mervaud, 2003), Voltaire and the 1760s: Essays for John Renwick (2008), et Cambridge Companion to Voltaire (2009).

nicholas.cronk@voltaire.ox.ac.uk

Résumés

Voltaire et le don du manuscrit

Voltaire a une manière toute particulière d'appréhender les relations complexes qui existent entre l'imprimé et le manuscrit dans la culture littéraire de l'Ancien Régime. En premier lieu, on trouve les manuscrits qui précèdent la première publication d'une œuvre sous forme imprimée et nous éclairent sur sa genèse, comme le manuscrit La Vallière de Candide, qui témoigne des différentes étapes par lesquelles est passé l'auteur pour terminer son roman. Par ailleurs, il existe des manuscrits qui remplacent l'œuvre imprimée. Dans certains cas - l'Épître à Uranie ou le Sermon des cinquante - Voltaire a délibérément choisi de faire circuler et de publier une œuvre manuscrite, plutôt que de l'imprimer. Enfin, on connaît des manuscrits qui viennent se superposer à l'œuvre imprimée : par exemple, une édition imprimée de Mahomet, conservée à la $\mathrm{BnF}$, contenant deux séries de notes manuscrites, la première de la main de Voltaire, la seconde de celle de César de Missy, qui collaborait à l'élaboration d'une édition londonienne de la pièce. Si ce document imprimé était en réalité un manuscrit de travail, d'autres, incluant des additions manuscrites, étaient à l'évidence destinés par Voltaire à être offerts à ses amis. Cet éventail d'exemples montre à quel point l'activité d'écriture, la production de manuscrits, est au centre de la créativité de Voltaire.

Voltaire has a unique appreciation of the complex relations linking print and manuscript in the literary culture of the Ancien Régime. Firstly, there are those manuscripts which predate the first publication of a work in print and which give us insight into the work's genesis. Thus the La Vallière manuscript of Candide provides some evidence of the stages in which Voltaire completed his novel. Secondly, there are manuscripts which replace the printed work. In such cases as the Épître à Uranie or the Sermon des cinquante, Voltaire chose deliberately to circulate and publish a work in manuscript form rather than publish it in print. Thirdly, there are examples of manuscript being superimposed on the printed work. A printed edition of Mahomet in the BnF, for example, contains two sets of manuscript notes, the first by Voltaire, and the second by César de Missy who was collaborating with him to prepare a London printing of the play. This printed item was in fact a working manuscript, but in other cases, printed works with manuscript additions were clearly intended by Voltaire as personal gifts to friends. This range of contrasting examples emphasises the extent to which the activity of writing, of producing manuscript, is central to Voltaire's creativity.
Voltaire hatte ein einzigartiges Verständnis der komplexen Beziehung von Druck und Manuskript in der literarischen Kultur des Ancien Régime. Zum einen gibt es diejenigen Manuskripte, die der ersten gedruckten Publikation vorausgehen, und die uns Einblick in die Entstehung des Werkes geben. Das La Vallière Manuskript von Candide liefert demnach Belege für die Stadien, in denen Voltaire seinen Roman fertigstellte. Zum zweiten gibt es auch Manuskripte, die das gedruckte Werk ersetzen. In Fällen wie Épître à Uranie oder der Sermon des cinquante entschied Voltaire absichtlich, ein Werk als Manuskript in Umlauf zu bringen, anstatt es gedruckt zu veröffentlichen. Zum dritten gibt es Beispiele von Handschriften, die dem gedruckten Werk zugefügt sind. So enthält eine gedruckte Ausgabe Mahomets der BnF zwei Gruppen von Manuskriptnotizen, die erste von Voltaire und die zweite von César de Missy, der mit Voltaire einen Londoner Druck des Stückes vorbereitete. Es handelte sich hier um ein Arbeitsmanuskript, aber in anderen Fällen intendierte Voltaire gedruckte Werke mit handschriftlichen Ergänzungen eindeutig als persönliche Geschenke an Freunde. Die Bandbreite unterschiedlichster Beispiele zeigt auf, wie zentral die Aktivität des Schreibens und der Manuskripterstellung für Voltaires Schaffensprozess waren.

Voltaire tiene una manera muy particular de aprehender las relaciones complejas que existen entre lo manuscrito y lo impreso en la cultura literaria del Antiguo Régimen. En primer lugar, se encuentran los manuscritos que preceden la primera publicación de una obra en forma impresa -los cuales nos informan sobre la génesis-, como el manuscrito La Vallière de Cándido, que muestra las diferentes etapas que ha atravesado el autor hasta terminar su novela. Por otro lado, existen manuscritos que reemplazan la obra escrita. En algunos casos -Epístola a Urania o El Sermón de los Cincuenta-, ha preferido deliberadamente hacer circular y publicar una obra manuscrita, en lugar de imprimirla. Finalmente, se conocen manuscritos que se superponen a la obra impresa: por ejemplo, una edición impresa de Mahoma, conservado en la Biblioteca Nacional de Francia, que contiene dos series de notas manuscritas, la primera de Voltaire y la segunda de César de Missy, quien colaboraba en la elaboración de una edición londinense de la pieza. Si este documento impreso era en realidad un manuscrito de trabajo, otros, incluyendo algunos añadidos manuscritos, estaban destinados por Voltaire, claramente, a ser regalos para sus amigos. Este abanico de ejemplos muestra hasta que punto la actividad de escritura, la producción de manuscritos, se sitúa en el centro de la creatividad de Voltaire. 
Voltaire esibisce una comprensione unica delle complesse relazioni che legano manoscritto e opera a stampa nella cultura letteraria dell'Ancien Régime. In primo luogo, troviamo i manoscritti che precedono la prima edizione a stampa di un'opera e che ne mostrano la genesi. Così, il manoscritto La Vallière del Candide offre prova degli stadi percorsi da Voltaire per completare il romanzo. In secondo luogo, ci sono i manoscritti che rimpiazzano l'opera a stampa. In questi casi, esemplificati da Épître à Uranie o dal Sermon des cinquante, Voltaire scelse deliberatamente di far circolare e pubblicare un'opera in forma manoscritta invece che a stampa. In terzo luogo, ci sono esempi di manoscritti sovrapposti all'opera stampata. Un'edizione del Mahomet conservata alla $\mathrm{BnF}$, per esempio, contiene due ordini di note manoscritte, il primo di Voltaire e il secondo di César de Missy, che collaborava con lui nella preparazione di una stampa della pièce teatrale a Londra. Questo testo stampato era, in realtà, un manoscritto di lavoro, mentre in altri casi opere a stampa con aggiunte manoscritte erano chiaramente intese da Voltaire come doni personali ad amici. Quest'ampiezza di casi diversi e contrastanti mette in luce quanto importante e centrale fosse per la creatività di Voltaire l'attività della scrittura e della stessa produzione di manoscritti.
Voltaire encara de forma extremamente original as complexas relações que se estabeleciam entre impresso e manuscrito na cultura literária do Antigo Regime. Primeiro, há os manuscritos que precedem a primeira publicação impressa de uma obra e nos oferecem uma visão íntima da sua génese. É o caso do manuscrito La Vallière de Candide, que fornece evidências dos sucessivos estágios do desenvolvimento da novela. Depois, há manuscritos que substituem a obra impressa, caso de Épitre à Uranie ou de Sermon des cinquante, em que Voltaire escolheu deliberadamente fazer circular e tornar públicos obras em manuscrito, em vez de as imprimir. Em terceiro lugar, há exemplos de manuscrito que é sobreposto ao texto impresso. Uma edição impressa de Mahomet na BnF, por exemplo, contém dois jogos de notas manuscritas, um de Voltaire e o outro de César de Missy, que com ele colaborava na impressão londrina da peça. Na realidade, este exemplar impresso não passava de um manuscrito em trabalho; em outros casos, livros impressos com adições manuscritas eram claramente preparados por Voltaire como ofertas pessoais a amigos. Esta gama de exemplos variados mostra até que ponto a actividade de escrever, de manuscrever, era central na creatividade de Voltaire. 